\title{
DESIGNING EFFECTIVENESS STUDIES OF INDIVIDUALIZED TREATMENTS WITH PERSONALIZED OUTCOMES
}

\author{
Johannessen $\mathrm{H}^{*}$, Agdal R*, Green A**, Hjelmborg J*, Lunde A*, Nissen $\mathrm{N}^{*}$, \\ Ostenfeld-Rosenthal A*, Pedersen $\mathrm{CG}^{* * *}$, Sørensen J*, Techau $\mathrm{M}^{*}$, Zachariae $\mathrm{R}^{* * *}$ \\ Department of Public Health, University of Southern Denmark*, \\ Institute of Clinical Research, University of Southern Denmark**, \\ Unit for Psychooncology and Health Psychology, Department of Oncology, Aarhus University Hospital and \\ Department of Psychology and Behavioral Science, Aarhus University, Denmark***
}

\section{Corresponding author:}

Helle Johannessen, professor

Department of Public Health, University of Southern Denmark,

JB Winslows Vej 9B, DK-5000 Odense C

Email: hjohannessen@health.sdu.dk

\begin{abstract}
Background: Patients with chronic or severe health problems or involved in rehabilitation programs often have needs that vary greatly. As a response to this, the health interventions are most often individualized and aimed at personalized outcomes.

Aim: The aim of this paper is to investigate how to design clinical trials that can measure the effectiveness of individualized interventions with personalized outcomes.

Methods: With departure in a discussion of pragmatic trials designed to assess outcomes of individualized treatments, further developments for acknowledging patients' personal priorities in outcomes are explored, including a discussion of randomization vs self-selection and presentation of flexible tools for the selection of personalized primary outcomes. Finally, the relevance of a cross-disciplinary approach is suggested.

Conclusion: Trials investigating the effectiveness of individualized interventions for personalized outcomes can be based on the design of pragmatic trials, with a few additional extensions. Tools for outcome must allow for personalized preferences and may include interviews, questionnaires with open formulation of concerns, or personal prioritizing of issues covered by validated questionnaires. In order to encompass patients' personal choice of outcomes, a cross- disciplinary methodology may be necessary.
\end{abstract}

Keywords: pragmatic trials, personalized medicine, randomization, self-select treatment, rehabilitation, patient preferences. 


\section{Introduction}

Individualized health interventions have become more common in conjunction with the growing number of persons suffering from chronic diseases, as well as, in relation to rehabilitation programs and adjunct treatments for persons suffering from severe illness. Patients with chronic or severe health problems or who are involved in rehabilitation programs often have resources and needs that vary greatly. As a response to this, the health interventions for them are most often individualized and aimed at personalized outcomes even though the patients suffer from identical diseases. There is therefore a need to investigate the effectiveness and the efficacy of individualized interventions with personalized outcomes.

Standard research designs for clinical trials are not well suited for interventions based on such double individualization, and the present article presents steps in a cross- disciplinary group working with development of design for trials that aim to evaluate personalized outcomes of indivi-dualized interventions. The quest takes departure in pragmatic trials designed to assess outcomes of treatments designed individually for each patient. In addition and in order to acknowledge patients' differing priorities in outcomes as well as in treatment, the choice between randomization vs. self-selection is discussed, and inclusion of flexible tools for the selection of personalized primary outcomes is proposed. Finally, the relevance of a cross-disciplinary approach is suggested in order to handle the wide range of potential primary outcomes that may be implied in studies acknowledging personal preferences of outcomes.

\section{The area of relevance}

In recent years we have witnessed a growing employment of individualized treatments; a trend that calls for evaluative procedures able to encompass the individualization of the treatment. For example, some forms of cancer treatment have increasingly turned towards therapeutic approaches targeting therapy for genetically defined subgroups of patients rather than for the disease. This implies that patients suffering from cancers of the same body site not necessarily receive identical interventions, but instead receive interventions that are aimed at particular and individual features ins the genetics of each patient. This kind of intervention is still in development but is seen by many as a promising path for cancer treatment in the future, and the methodology for investigating the effectiveness and efficacy of the individualized interventions is being discussed and developed (Freedman et al, 2010; Henderson \& Schumacher, 2011).

Also in relation to chronic and lifestylerelated diseases as well as rehabilitation from severe illness, healthcare interventions tailored to the individual patient is being called for. In this context, the aim of the intervention is to enable the individual to live a fulfilling life with a disease, or to rehabilitate from severe illness, and the outcome of the intervention often concerns psychosocial aspects related to everyday life. While the individualized genomic cancer 
treatments, as mentioned above, seek to cure the patients from the cancer and thus have identical outcomes in spite of individualized interventions, interventions for chronic diseases or within rehabilitation often imply that the desired results of the treatment differ from one person to another. The aims of patients with such health problems may be as divergent as to be free of pain, to attain increased quality of life, to return to work, to sleep better, etc.. This has created a demand for research methodologies suited not only to encompass individualized interventions but also personalized out-comes.

Our aim has been to reflect on what is necessary for valid and reliable research assessing the effectiveness of individualized interventions that have personalized out-comes.

\section{Pragmatic trials as a starting point for individualized treatments}

The design of the pragmatic trial has been developed over the past decades as an approach to determine the effects of an intervention under the usual conditions in which it will be applied (Thorpe et al, 2009). It has been argued that the design of this approach departs from the design for explanatory trials in certain welldefined ways. In a classic text from 1967, Schwartz and Lellouch coined the terms "pragmatic" and "explanatory" trials in order to distinguish between trials designed to answer two different research questions. While the pragmatic trial is well suited to establish knowledge that can help choose between options for care, the explanatory trial is well suited for testing causal research hypotheses, for example that a particular intervention causes a particular biological change (Schwartz \& Lellouch, 2009; Zwarenstein et al., 2008). In other words, the explanatory trial aims to test a hypothesis of the effect of a particular anticipated active ingredient in order to contribute to the understanding and explanation of the treatment efficacy, while pragmatic trials concern whether the therapy is effective in practice as usual without considering the active ingredients or working mechanisms (Zwarenstein et al. 2008).

A major difference between the two ideal trial designs is that the pragmatic trial is designed to test the effectiveness of clinical interventions applied in usual healthcare practice that are flexible and tailored to the individual patient. The explanatory trial, on the other hand, tests the efficacy of a particular treatment applied under ideal conditions and is characterized by a strictly enforced treatment regime and a selection of patients that meet stringent and well defined criteria for inclusion. For both types of trial it is of importance to describe the interventions applied; but for the pragmatic trials, the description must reach beyond the technique applied as the feasibility and success of an intervention may also depend on attributes of the healthcare system and the setting in which the trial takes place. Information regarding the wider setting thus becomes necessary for an assessment of the relevance and applicability of the results in other, possibly different, settings (Zwarenstein et al., 2008). Further, explanatory trials usually focus on relatively homogenous patient groups, while the patient group in pragmatic 
trials often will be heterogeneous. The heterogeneity of the patient group will often imply that a larger sample size is needed than in an explanatory trial in order to level out the variations in background and disease pattern among patients (Macpherson, 2004).

Another main difference between ideal explanatory and pragmatic trials is that the latter has declined on the demand of blinding. Blinding is closely linked to the question of working mechanisms as it is a means to investigate whether a given change in health status is caused by the therapy itself or other "non-specific" factors, e.g. the patients' expectations or effectiveness as modifying factors (Zwarenstein et al., 2008). attitude towards the therapy. As pragmatic trials do not address questions of specificity, but solely focus on whether the intervention has an effect or not, blinding of patients and practitioners is not necessary although it is desirable and often possible to blind the assessor or obtain an objective source of data for evaluation of outcomes. In pragmatic trials, issues such as subjective attitudes of belief or disbelief in the intervention, enthusiasm, effort and degree of optimism in the self-assessment of outcomes may add to (or detract from) the effects of an intervention, and it may thus be valuable to incorporate these issues in the estimate of The pragmatic trial is designed to take

Table 1. Key domains that distinguish pragmatic from explanatory trials

1. The eligibility criteria for trial participants.

2. The flexibility with which the experimental intervention is applied.

3. The degree of practitioner expertise in applying and monitoring the experimental intervention.

4. The flexibility with which the comparison intervention is applied.

5. The degree of practitioner expertise in applying and monitoring the comparison intervention.

6. The intensity of follow-up of trial participants.

7. The nature of the trial's primary outcome.

8. The intensity of measuring participants' compliance with the prescribed Intervention, and whether compliance improving strategies are used.

9. The intensity of measuring practitioners' adherence to the study protocol, and whether adherence-improving strategies are used.

10. The specification and scope of the analysis of the primary outcome.

(Thorpe et al., 2009) 
the heterogeneity of everyday clinical intervention into account, while still aligning with the explanatory trial with respect to the need for control groups for comparison of outcomes. The pragmatic trial, however rarely uses placebo treatment as control condition, as is usual in explanatory trials. Instead, pragmatic trials often compare new interventions to "treatment as usual" or "usual care" in order to increase the applicability and feasibility of the intervention (Zwarenstein et al., 2008).

\section{A pragmatic-explanatory continuum indicator}

Although explanatory and pragmatic trials were originally proposed as two mentioned above. However, in order distinct types of design for the evaluation of the effect of an intervention (Gartlehner, Hansen, Nissman, Lohr, \& Carey, 2006; Schwartz \& Lellouch, 2009), it has been argued, that they should be seen as ideal types of design, and that actual trials most often will be positioned in between the two (Thorpe et al., 2009). In this perspective, the two types of design are not seen as distinct (and clean) trials, but merely as two ends of a continuum in which actual trials will be positioned according to the choices within ten domain (see Table 1).

Thorpe et al (2009) proposes that the ten domains are used to assess the specific characteristics of a trial and has developed the list into a tool (the pragmatic-explanatory continuum indicator summary (PRECISE tool)) illustrated as a wheel in order to get a plot that makes sense intuitively and can be used without special training (see Figure 1). The wheel depicts whether at trial tends to take a broad (pragmatic) or a narrowly focused (explanatory) view of the intervention. The broader the view, the more flexibility will be involved in the trial set-up.

The ideal explanatory trial will be positioned close to the center, while the ideal pragmatic trial will be positioned at the end of each of the spokes of the wheel, but many projects will be positioned at various points along the spokes (see Thorpe et al. 2009 for an elaboration of this).

A trial of individualized therapy with personalized outcomes aligns with the pragmatic trial with respect to most of the requirements to meet the challenge of testing effects on personalized outcomes, the design must be reconsidered in regard to the choice of primary outcomes, and it may have to be reconsidered in regard to randomization and types of control groups, which we will discuss below.

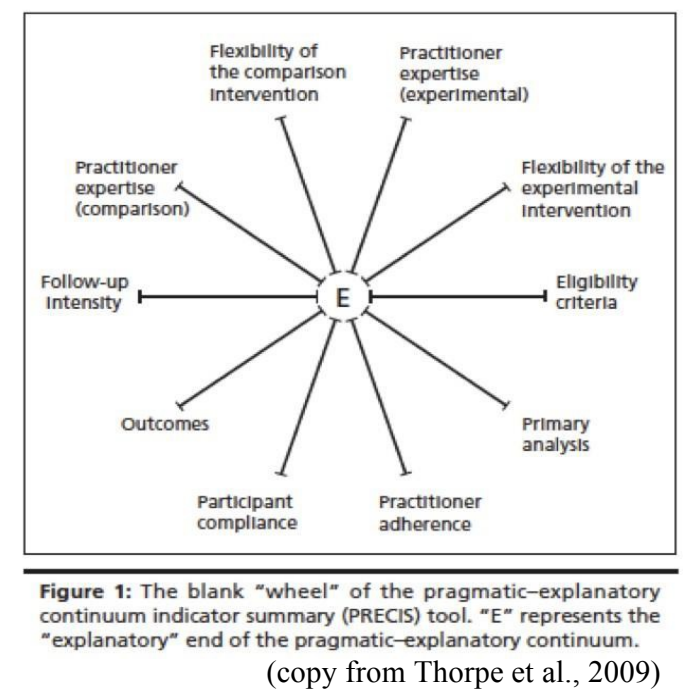




\section{Focus on personal preference}

The above concerns methodological implications of studying the outcomes of interventions that are individualized. But our aim concerns trials that not only allow for individualization of the intervention but also of the outcomes, and for that purpose it is necessary to expand the discussion to also cover personal choice of primary outcomes. In order to make such a design suitable for trials of interventions in which randomization is not always preferable (for example in palliative care, or in certain forms of rehabilitative activities) it may also be relevant to consider implications of randomization versus patient preference for intervention. In Table 2, the expansions needed in relation to traditional pragmatic trials are listed, and in the following, these issues will be discussed.

\subsection{Randomization or patient preference}

The first issue where trials with personalized outcomes may differ from ordinary pragmatic trials concerns whether participants must be randomized to intervention or control group, or whether it is feasible to let patients choose themselves whether they find the intervention on trial relevant for themselves. The PRECIS tool developed by Thorpe et al (2009) grew from the Pragmatic Randomized Controlled Trials in Health Care, and even though they do not specify the need for randomization in their paper, one can antipate that they anticipate a trial is randomized when taking into consideration this base of the initiative. The question of whether to randomize or not may be irrelevant if the intervention aims at cure of a disease, but if it concerns rehabilitation or palliative care, for example, it may be very relevant to take into consideration whether the therapeutic intervention may be of personal relevance for the patients involved. For many years, randomization has been a core requirement in clinical trials in order to avoid systematic errors, e.g. bias due to self-selection or sampling of particularly well-disposed patients to the intervention group. Patients subjective attitudes towards an intervention are known to influence the results of an intervention, and it has for long been anticipated that if patients self-select their participation, personal preferences may compromise the internal validity of a study.

In a systematic review of the influence of self-selection versus randomization of participants (King et al., 2005), it is, however, concluded that self-selection can be applied in investigations of the effectiveness of therapeutic interventions when randomization is unacceptable to clinicians and/or patients and their families. By allowing for self-selection external validity and participation rates may be maximized. When comparing results of studies that were based on randomization with results from studies that included selfselection for treatment, it was concluded that the outcomes for self-selecting patients are similar to those obtained in randomized trials when the exclusion criteria are carefully defined and when the prognostic factors and the preferences of patients and professionals are well understood (King 
Table 2. Key differences between explanatory and pragmatic trials - with extensions for personalized outcome preferences

\begin{tabular}{|c|c|c|c|}
\hline & Explanatory trial & Pragmatic trial & Pragmatic trial including personalized outcomes \\
\hline Question & Efficacy - is the intervention effective? & $\begin{array}{l}\text { Effectiveness - is the intervention effective when } \\
\text { used in normal practice? }\end{array}$ & $\begin{array}{l}\text { Effectiveness - is the intervention effective according } \\
\text { to patient preferences for outcomes when used in } \\
\text { normal practice }\end{array}$ \\
\hline Setting & $\begin{array}{l}\text { Well resourced, "ideal" setting } \\
\text { Experimental setting }\end{array}$ & $\begin{array}{l}\text { Normal practice } \\
\text { Routine care setting }\end{array}$ & $\begin{array}{l}\text { Normal practice where individualized preferences for } \\
\text { intervention and outcomes are natural (e.g. } \\
\text { rehabilitation, CAM and psychosocial interventions) }\end{array}$ \\
\hline Participants & $\begin{array}{l}\text { Highly selected. Poorly adherent participants and } \\
\text { those with conditions which might dilute the effect } \\
\text { are often excluded. } \\
\text { Homogenous patients blinded to minimize bias, may } \\
\text { manage with smaller sample sizes }\end{array}$ & $\begin{array}{l}\text { Little or no selection beyond the clinical indication of } \\
\text { interest } \\
\text { Heterogeneous patients unblinded to maximize } \\
\text { synergy, may need larger sample sizes }\end{array}$ & $\begin{array}{l}\text { As for pragmatic trials - but patients may be allowed } \\
\text { to choose between intervention and control groups in } \\
\text { order to maximize participation }\end{array}$ \\
\hline Intervention & $\begin{array}{l}\text { Strictly enforced and adherence is monitored closely } \\
\text { Standardized treatment, placebo controlled, aim to } \\
\text { equalize non-specific effects, simple interventions, } \\
\text { usually short-term follow-up. } \\
\text { Practitioner skilled for standard protocol }\end{array}$ & $\begin{array}{l}\text { Applied flexibly as it would be in normal practice } \\
\text { Not placebo controlled, aim to optimize non-specific } \\
\text { effects, complex interventions, often long-term } \\
\text { follow-up. } \\
\text { Practitioner skilled in routine care }\end{array}$ & As for pragmatic trials \\
\hline Outcome & Often short term surrogates or process measures & $\begin{array}{l}\text { Directly relevant to participants, funders, } \\
\text { communities, and healthcare practitioners }\end{array}$ & $\begin{array}{l}\text { Personalized selection of primary outcomes - either } \\
\text { personally defined outcomes or personal priority of } \\
\text { main outcome areas }\end{array}$ \\
\hline Analysis & $\begin{array}{l}\text { An intention-to-treat analysis is usually performed for } \\
\text { all patients. However, this may be supplemented by a } \\
\text { per-protocol analysis or an analysis restricted to } \\
\text { "compliers" or other subgroups in order to estimate } \\
\text { maximum achievable treatment effect. Analyses are } \\
\text { conducted that attempt to answer the narrowest, } \\
\text { "mechanistic" question (whether biological, } \\
\text { educational or organizational) }\end{array}$ & $\begin{array}{l}\text { The analysis includes all patients regardless of } \\
\text { compliance, eligibility, and others (intention-to-treat } \\
\text { analysis). In other words, the analysis attempts to see } \\
\text { if the treatment works under the usual conditions, } \\
\text { with all the noise inherent therein }\end{array}$ & $\begin{array}{l}\text { As for pragmatic trials - } \\
\text { Due to the variety in patient chosen outcomes, a } \\
\text { cross-disciplinary approach may be needed }\end{array}$ \\
\hline $\begin{array}{l}\text { Relevance to } \\
\text { practice }\end{array}$ & $\begin{array}{l}\text { Indirect - little effort made to match design of trial to } \\
\text { decision making needs of those in usual setting in } \\
\text { which intervention will be implemented (Low } \\
\text { relevance/impact on practice) } \\
\text { High internal validity }\end{array}$ & $\begin{array}{l}\text { Direct - trial is designed to meet needs of those } \\
\text { making decisions about treatment options in settings } \\
\text { in which intervention will be implemented (High } \\
\text { relevance/ impact on practice) } \\
\text { High external validity }\end{array}$ & $\begin{array}{l}\text { As for pragmatic trials - } \\
\text { But specifically aimed at healthcare where a single } \\
\text { reliable/robust study outcome is not expected } \\
\text { High external validity }\end{array}$ \\
\hline
\end{tabular}


et al., 20015). This extended view of what is necessary to assess at baseline indicates the necessity of a thorough preexamination of participants.

Given the increased external validity associated with higher participation rates, it may be preferable to avoid randomization when the treatment involves active participation of the patients in actions that are unusual or in contrast to ordinary everyday activities or personal beliefs. This could be the case in treatments based on repeated physical exercises (e.g. gymnastics, swimming, or more alternative ways of movement such as yoga and qigong) for persons that are not habitually doing physical exercise in their everyday life. Or it could be relevant in case of cultural aspects that are contrary to everyday experience and therefore may appear meaningless to some patients. e.g. trials of therapies based on spiritual prior to the first contact to patients. In this design, a random collection of patients would be allowed to choose between the intervention group and the control group. Another and similarly random group of patients would be randomized to intervention and control, and the two intervention groups could be compared with respect to participation rates and outcome scores. In a recent study the authors behind this article tried this overall design in a trial of energy healing as rehabilitation after colorectal cancer, and although the selfselect treatment group filled up a little faster than the rest of the groups, it turned out not to be a problem to fill the self-select control group; the reason for persons to elect to be in the control group appeared to be a wish to serve assumptions to patients raised in a secularized socio-cultural environment, or trials based on body-images that are unfamiliar to the patients and contrary to their habitual ways of conceptualizing the body.

In order to secure not only external validity but also high internal validity in a study based on patient preference for intervention or control group, rather large sample sizes are necessary, and the analysis of outcomes must account for baseline measures of the same parameters as those chosen as outcomes (King et al., 2005).

One way of investigating whether personal preferences has an impact on participation rates and outcome scores and at the same time include randomization of all participants, in order to avoid selection bias, could be to randomize all participants to selfselection and randomization science, but not having time or need for treatment (see box 1).

\subsection{Personalized outcomes}

While pragmatic trials ideally rely on outcome measures "unarguably important such as mortality and severe morbidity" (Zwarenstein et al., 2008), this may be difficult in regard to therapies that are not aimed at curing a disease but at helping people return to a normal everyday life. For this reason, it has been suggested to choose outcome measures that are directly relevant to participants, funders, communities, and healthcare practitioners (Zwarenstein et al.,

2008). In order to ensure the relevance of the outcomes for the 
patients in cases where it is not cure of a disease that is at stake, the outcome measures must include some assessment of the personal preferences for various types of outcome. On the other hand, and in order to ensure general relevance for research and a wider community of patients and practitioners, the outcomes must be standardized and measured by validated tools.

To establish this dual perspective, we suggest to let participants themselves select preferred outcome goals and at the same time to use standardized and validated tools (i.e. validated questionnaires and visual analogue scales, various physical measurements, etc.). In order to let participants choose preferred outcome goals, we suggest implying methods that either let participants formulate in their own words their hopes and expectations for the outcomes prior to the treatment, and perhaps also at certain prefixed times later in the course in order to be sensitive to changes in desired outcomes if they should occur. Available tools to let participants formulate in their own words their desired outcome goals include interviews and the MYMOP and the MYCaW questionnaires (Paterson, Thomas, Manasse, Cooke, \& Peace, 2007). The experienced effectiveness can be reported by participants in words or on visual analogue scales, reflecting to what extend the particular issue concerns them before and after the intervention. Alternatively, or in combination, participants could be asked to prioritize a number of preselected outcome measures according to how important they find these to be for them. These outcome measures should refer to particular included validated questionnaires or measurements, and the participant chosen measurements could be considered as primary outcomes allowing for personal preferences of outcomes although the tools used to measure them are standardized and validated (see box 1).

The three approaches to catching personalized outcomes could be visualized as positions on a continuum between free formulation at the one end and choice between preselected categories of concerns at the other end, with the interview as the approach offering an opportunity for the most free formulation, and the priority list being most pre-selected; while the MYMOP and MYCaW questionnaires offer the opportunity for free formulation of concerns but implies subsequent classification of concerns by the researchers (Ostenfeld- Rosenthal \& Johannessen, 2014).

\subsection{Pros and cons of cross-disciplinary approaches}

To secure openness to patient preferences regarding outcomes and at the same time establish valid and reliable evaluation of these, it is clearly an advantage to have several methodologies at your disposal. Which methodologies that will be relevant depend on the kinds of outcomes chosen and on the research perspective of effectiveness (quantitative or qualitative analysis). It may thus be necessary to draw on methods and tools from 
different methodologies and disciplines in order to measure items that e.g. regard the severity of particular health problems, the economy of the intervention, or participants' habits, values and health seeking behavior. If the trial involves patient preferences of outcomes, it is important to collect baseline information on preferences, as well as, previous experiences with and prevailing expectations to the therapy.

In a paper discussing designs of mixed method studies, the authors suggest several models for the organization of interdisciplinary collaboration (Creswell et al., 2004). We suggest that projects investigating the effect of a treatment in regard to patient preferences for outcomes, pay attention to models that allow a number of different methodologies and research traditions to be of equal importance from the outset, as the relative importance of each approach will be determined by the participants' choice of primary outcomes. For this purpose, The Triangulation Design Model seems relevant. In this model, several kinds of data are collected with different methodologies at the same time, and in the analysis and presentation of results they supplement each other (Creswell et al., 2004). If we acknowledge the patients' preferences for outcomes and divide the dataset into subgroups based on preferences for outcomes, the analysis of data regarding each subgroup may require the skills of a particular discipline. That implies that even though all disciplines are equal in the overall project, they may each become primary in regard to specific subgroups of the dataset. In our study, see Box 1, we did for example employ a psychologist to deal with data regarding psychological factors (e.g. quality of life, mood), a biologist to deal with data regarding physical activity, anthropologists to investigate patients subjective experiences and a health economist to deal with data regarding cost-effectiveness. An issue of importance when engaging in crossdisciplinary or cross-methodological research on effectiveness is that different methodologies imply different time scales in terms of data generation and analysis. An approach based on interviews and participant-observation will, in example, generate and analyze data all along the research process in order to build the dialogue in one interview upon the dialogue and information gathered previously. In questionnaire studies, it is however not appropriate to start the data analysis until all questionnaires have been compiled. Such differences in the perspectives of time within a project may hinder ongoing synergy between the different approaches.

\section{Conclusion}

We suggest that trials investigating personalized outcomes of individualized interventions take departure in the design of what have been called pragmatic trials, with a few additional extension (see Table 2). Of main importance is that the intervention is flexible and able to meet the needs of each patient, and that tools for outcome measurement allow for personalized preferences in outcomes. Additionally, it may be relevant to allow patients to 
choose whether they want to be in the intervention or the control group. In order to encompass patients' personal choice of outcomes, a cross-disciplinary approach may be necessary.

\section{Box 1. Design of a trial with personalized outcomes}

In a recent pragmatic trial on energy healing as rehabilitation after hospital treatment for colorectal cancer (Ostenfeld-Rosenthal \& Johannessen, 2014; Pedersen, Johannessen, Hjelmborg, \& Zachariae, 2014; Techau et al., 2014), the design of a pragmatic trial was elaborated with the aim to maximize adherence (allow for selfselection) and to let participants themselves choose which outcome they wished to be primary.

\section{Randomization or self-selection}

A total of 248 persons identified through a national patient registry agreed to participate in the trial. Prior to the first contact, participants were randomized into two groups: One to be further randomized to treatment or control, and one to be offered to selfselect whether they wanted to be in the control group or in the treatment group. The self-select treatment group had more participants (82 participants) than any of the randomized groups (55-58 participants), but the self-select control group was easily populated and similar to the randomized groups in size (52 participants). The participants in the self-select treatment group were younger, better educated and with a higher proportion of women, than participants in the other groups. When adjusting for age, education and gender, no difference in outcomes were detectable between randomized and self-select treatment groups. The overall response rate was, however, higher in the self-select group (95\% returned questionnaires after six weeks) than in the randomized group $(80 \%$ returned questionnaires after six weeks).

\section{Choice of personalized outcomes}

Two methods were employed to let participants choose themselves the outcomes that were of most importance for them. Both methods were questionnaire based and employed simultaneously to participants.

In one method (the MYCaW questionnaire) participants formulated 1-2 concerns they hoped to improve at baseline and again after 3 weeks. The participants wrote the concerns in open slots of a questionnaire and scored each of them on a VAS-scale $(0=$ does not bother me at all, $6=$ =bothers me a great deal). The concerns formulated by participants were repeated on follow-up questionnaires by the researchers for repeated scoring. Although the concerns were subsequently categorized in order to get an overview of what kinds of concerns participants had stated, the scoring was calculated for each group of participants (treatment or control groups) as a whole. The aim was not to investigate whether the treatment would help to improve specific 
concerns, but to test whether it could help improve whatever concerns the participants had expressed.

The other method consisted of a list of themes covered by validated questionnaires in the package of questionnaires distributed to all participants; the list was constructed by the research team. All participants were asked to choose the most important area of concern on a priority list that presented seven overarching categories of concerns covered by the validated questionnaires: Cancer related symptoms (including late effects of cancer treatment), physical activity, quality of life, depressive symptoms, mood, sleep quality, economy. A further option, 'other concerns', was included in the priority list. The aim was to identity which of the validated questionnaires that should be considered as primary outcome measure for each of the participants.

\section{Conflict of interest}

None to declare.

\section{Acknowledgements}

Financial support was provided by The Danish Council for Strategic Research, Ministry of Science, Innovation and Higher Education (Grant no. 09-065176); Department of Psychology and Behavioral Sciences, University of Aarhus; and the Institute of Clinical Research and Department of Public Health, University of Southern Denmark. The funding sources had no involvement in the study design, collection, analysis, and interpretation of data, or writing of the paper. 


\section{References}

Creswell JW, Fetters MD, Ivankova NV. (2004). Designing a mixed methods study in primary care. Annals of Family Medicine, 2(1), 7-12.

Freedman AN, Sansbury LB, Figg WD, Potosky AL, Weiss Smith, SR, Khoury MJ, Minasian LM. (2010). Cancer pharmacogenomics and pharmacoepidemiology: setting a research agenda to accelerate translation. J Natl Cancer Inst, 102(22), 1698-1705.

doi: $10.1093 /$ jnci/djq390

Gartlehner G, Hansen RA, Nissman D, Lohr KN, Carey TS. (2006). A simple and valid tool distinguished efficacy from effectiveness studies. $J$ Clin Epidemiol, 59(10), 1040-1048.

doi: 10.1016/j.jclinepi.2006.01.011

Henderson R, Schumacher M. (2011). Clinical epidemiology and individualized medicine. Biom J, 53(2), 167169.

doi: $10.1002 / \mathrm{b} \mathrm{imj} .201000257$

King M, Nazareth I, Lampe F, Bower P, Chandler M, Morou M, . . . Lai R. (2005). Conceptual framework and systematic review of the effects of participants' and professionals' preferences in randomised controlled trials. Health Technology Assessment, 9(35).

Macpherson H. (2004). Pragmatic clinical trials. Complementary Therapies in Medicine, 12(2-3), 136-140.

Ostenfeld-Rosenthal A, Johannessen $\mathrm{H}$. (2014). How to capture patients' concerns and related changes: Comparing the MYCaW questionnaire, semi-structured interview and a priority list of outcome areas. Complementary Therapies in Medicine, 22(4), 690-700.

doi:10.1016/j.ctim.2014.06.009
Paterson C, Thomas K, Manasse A, Cooke H, Peace G. (2007). Measure Yourself Concerns and Well being (MYCaW): An individualised questionnaire for evaluating outcome in cancer support care that includes complementary therapies. Complementary Therapies in Medicine, 15(1), 38-45.

doi: http://dx.doi.org/10.1016/j.ctim.2006. 03.006

Pedersen CG, Johannessen H, Hjelmborg JvB, Zachariae R. (2014). Effectiveness of energy healing on Quality of Life: a pragmatic intervention trial in colorectal cancer patients. Complementary Therapies in Medicine, 22(3), 463-472.

doi: 10.1016/j.ctim.2014.04.003

Schwartz D, Lellouch J. (2009) (originally 1967). Explanatory and pragmatic attitudes in therapeutical trials. $J$ Clin Epidemiol, 62(5), 499-505.

doi: 10.1016/j.jclinepi.2009.01.012

Techau M, Lunde A, Pedersen CG, Green A, Johannessen H, Nissen N. (2014). Nonparticipants and reasons for Nonparticipation in a pragmatic trial of energy healing as cancer rehabilitation. European Journal of Integrative Medicine, 6, 268276.

Thorpe KE, Zwarenstein M, Oxman AD, Treweek S, Furberg CD, Altman DG, ... Chalkidou K. (2009). A pragmaticexplanatory continuum indicator summary (PRECIS): a tool to help trial designers. CMAJ, 180(10), E47-57.

doi:10.1503/cmaj. 090523

Zwarenstein M, Treweek S, Gagnier JJ, Altman DG, Tunis S, Haynes B, ... Moher D. (2008). Improving the reporting of pragmatic trials: an extension of the CONSORT statement. BMJ, 337. doi:10.1136/bmj.a230 